\title{
EVALUACIÓN EN 18 HORAS DE LA DINÁMICA CARDIACA CON LA LEY MATEMÁTICA DE LOS SISTEMAS DINÁMICOS
}

\author{
EVALUATION IN 18 HOURS OF CARDIAC DYNAMICS WITH THE MATHEMATICAL LAW OF DYNAMIC \\ SYSTEMS
}

\author{
Signed Esperanza Prieto Bohorquez ${ }^{1,2}$, Javier Rodríguez Velásquez ${ }^{3}$, Catalina Correa Herrera ${ }^{4}$, Juan \\ Pardo $^{5}$, Javier Ardila.
}

\section{Resumen:}

Introducción: una ley exponencial se ha hallado para los sistemas dinámicos caóticos cardiacos, logrando cuantificar las diferencias entre dinámicas cardiacas normales y patológicas.

Metodología: Se analizaron 120 registros electrocardiográficos, 40 correspondían a sujetos dentro de los límites de normalidad y 80 con diferentes patologías. Para cada holter se analizaron los atractores generados con los datos durante 18 horas y durante toda la dinámica. Se calculó la dimensión fractal del atractor y su ocupación espacial. A estas medidas se aplicó la evaluación matemática diagnostica desarrollada previamente, comparando la evaluación para 18 horas y para todo el registro; finalmente se calculó la sensibilidad, especificidad y coeficiente Kappa.

Resultados: Para las dinámicas normales los espacios de ocupación en la rejilla Kp estuvieron entre 200 y 381 en la evaluación de la totalidad del holter, y entre 201 y 384 en la evaluación durante 18 horas, mostrando la cercanía en las medidas, lo que permite que la disminución en el tiempo de la evaluación sea consistente, esta misma cercanía se observó para las dinámicas enfermas y agudas.

Conclusión: Se evidenció la aplicabilidad clínica en 18 horas de la ley exponencial en la dinámica cardiaca caótica asociada a arritmias mostrando ser de utilidad para la predicción de la evolución hacia estados agudos de la dinámica.

Palabras clave: caos; fractal, ley; ley caótica; dinámica cardiaca; diagnóstico; arritmia

\section{Abstract:}

Introduction: an exponential law has been found for chaotic dynamic cardiac systems, making it possible to quantify the differences between normal and pathological cardiac dynamics.

Methodology: 120 electrocardiographic records were analyzed, 40 corresponded to subjects within the limits of normality and 80 with different pathologies. For each holter the attractors generated with the data during 18 hours and throughout the dynamics were analyzed. The fractal dimension of the attractor and its spatial occupation were calculated. To these measures was applied the diagnosis mathematical evaluation previously developed, comparing the evaluation for 18 hours and for the whole registry; sensitivity, specificity and Kappa coefficient were finally calculated.

Results: For the normal dynamics, the occupancy spaces in the Kp grid were between 200 and 381 for the evaluation of the whole holter, and between 201 and 384 in the evaluation during 18 hours, showing the closeness in the measurements, which allows that the decrease in the time of the evaluation is consistent, this same proximity was observed for the diseased and acute dynamics.

Conclusion: It was evidenced the clinical applicability in 18 hours of the exponential law in the chaotic cardiac dynamics associated with arrhythmias showing to be useful for the prediction of the evolution towards acute states of the dynamics.

Keywords: chaos; fractal; law, chaotic law; cardiac dynamics; diagnosis; arrhythmia

\footnotetext{
${ }_{1}^{1}$ Investigadora Grupo Insight. Centro de Investigaciones Clínica del Country, Bogotá D.C., Colombia.

${ }^{2}$ Email de contacto: grupoinsight2025@yahoo.es

${ }^{3}$ MD. Director del Grupo Insight. Centro de Investigaciones de la Clínica del Country. Cra. 79B № 51-16 Sur. Int. 5. Apto. 102, Barrio Kennedy, Bogotá D.C., Colombia. Tel: (57)(1)4527541;

${ }^{4}$ Psy Investigadora Grupo Insight. Centro de Investigaciones Clínica del Country, Bogotá D.C., Colombia.

${ }^{5}$ Cardiólogo. Director del Programa de Cardiología, Fundación Clínica Abood Shaio. Profesor de la Universidad del Bosque.

${ }^{6}$ MD. Ginecobstetra. Director del Departamento de Salud Reproductiva del Hospital Universitario Mayor Méderi y de la Universidad El Rosario.
} 


\section{Introducción}

La teoría de los sistemas dinámicos sirve para entender cómo cambian los procesos de la naturaleza, partiendo de la evolución temporal de las variables dinámicas ${ }^{(1,2)}$ de un sistema el comportamiento del sistema es determinado, a partir de esta teoría se han logrado importantes descubrimientos, como la existencia de caos. A partir de representaciones de las variables dinámicas del sistema en el espacio de fase es posible estudiar el comportamiento del sistema, estas representaciones son denominadas atractores, para sistemas aleatorios ${ }^{(3)}$ se obtienen atractores caóticos que presentan una forma irregular.

Los objetos irregulares han sido estudiados desde el siglo XIX, sin embargo solo a finales del siglo XX cobran importancia con la aparición de la geometría fractal, desarrollada por Mandelbrot. Esta geometría estudia los objetos irregulares denominados fractales, los cuales se caracterizan por autosemejanza, es decir, invarianza de escala. Los objetos naturales son fractales ${ }^{(4-6)}$, y en particular las formas de los atractores caóticos cardiacos también.

Las enfermedades cardiovasculares (ECV) representan el $1,9 \%$ de las muertes al año en las Américas, se estima que el $25 \%$ de las personas padecen de este tipo de enfermedad, y se calcula que pueden llegar a morir alrededor de 23.6 millones de personas con esta condición en el año $2030^{(7)}$. Para el 2011 , se registraron 229.6 muertes por cada 100.000 personas atribuidas a $\mathrm{ECV}^{(8)}$. Y se estima que continuará siendo la principal causa de muertes a nivel mundial.

El Holter es uno de los exámenes de diagnóstico más importante para identificar cambios transitorios significativos del ritmo cardiaco, de aparición súbita y asintomáticos ${ }^{(9)}$. Este examen permite registrar el tiempo $\mathrm{R}-\mathrm{R}$, lo que permite analizar la variabilidad de la frecuencia cardiaca $^{(10)}$, la aparición de episodios arrítmicos no perjudiciales post-infarto ${ }^{(11)}$ y arritmias como medida de deterioro vascular.

A partir de la teoría de los sistemas dinámicos se desarrolló una concepción normalidad enfermedad que revalúa la noción tradicional homeostática de normalidad, pues mientras que en esta se considera que la normalidad corresponde a la regularidad, la nueva concepción sostiene que una dinámica será enferma si es muy regular 0 altamente aleatoria $^{(12)}$ y es normal si está entre estos extremos, desde modelos matemáticos se han desarrollado metodologías de análisis de la dinámica cardiaca $^{(13-19)}$, por su variedad se discute cuáles de estos métodos deben aplicarse $^{(20)}$, así como su aplicabilidad ${ }^{(21)}$.

La aplicación de teorías físicas y matemáticas al estudio de la dinámica cardiaca permitió desarrollar una metodología basada en una ley matemática para los sistemas dinámicos caóticos cardiacos $^{(22,23)}$, la cual se ha aplicado a arritmias, así como a la dinámica de marcapasos, permitiendo establecer cuantificaciones y predicciones objetivas mediante los espacios de ocupación en el contexto de la ley. Recientemente se ha reducido el tiempo de análisis de la dinámica cardiaca gracias al carácter matemático de esta ley, con base en esto, la presente investigación busca analizar dinámicas cardiacas durante 18 horas con el fin de comprobar su capacidad de evaluación al disminuir el tiempo de 24 a 18 horas.

\section{Métodos}

\section{Definiciones}

Mapa de retardo de frecuencias cardiacas: Grafico bidimensional, en cada uno de los ejes se encuentran las frecuencias cardiacas y cada punto en el plano está constituido por el valor de una frecuencia y la frecuencia previa en el tiempo.

\section{Dimensión Fractal de Box-Counting (D):}

$$
\begin{gathered}
D=\frac{\log N\left(2^{-(K+1)}\right)-\log N\left(2^{-K}\right)}{\log 2^{k+1}-\log 2^{k}}=\log _{2} \frac{N\left(2^{-(k+1)}\right)}{N\left(2^{-k}\right)} \\
\text { Ecuación } 1
\end{gathered}
$$

Donde $N\left(2^{-k}\right)$ corresponde al número de cuadros ocupado por el objeto para la rejilla con partición $2^{-k}$.

Ley exponencial de la dinámica cardiaca caótica: Tomando la ecuación 1 y denominando los valores que toma la función $N\left(2^{-k}\right)$, como $\mathrm{Kp}$, para la rejilla con la partición más pequeña, y Kg, para la partición más grande:

$$
\begin{gathered}
D=\log _{2} \frac{N\left(2^{-(k+1)}\right)}{N\left(2^{-k}\right)}=\log _{2} \frac{K_{p}}{K_{g}} \\
\text { Ecuación } 2
\end{gathered}
$$

Se puede obtener una relación exponencial entre los espacios ocupados y la dimensión fractal (D):

$$
K_{p}=K_{g} 2^{D}
$$




\section{Población}

Se tomaron en total 120 registros electrocardiográficos 0 Holter de mínimo 21 horas, de los cuales 40 correspondieron a casos diagnosticados por un cardiólogo experto como normales, y 80 casos correspondieron a casos diagnosticados con diferentes tipos de patologías pertenecientes a sujetos mayores de 21 años.

\section{Procedimiento}

Se tomó la frecuencia cardiaca mínima y máxima, y el número de latidos en cada hora de sólo 18 horas para cada registro. Con estos datos se simularon las secuencias de valores de la frecuencia cardiaca, las cuales fueron graficadas en el espacio de fases mediante un mapa de retardo construyendo el atractor caótico (figura 1 y 2) de la dinámica cardiaca. Luego se procedió a calcular la dimensión fractal aplicando el método de Box Counting, el cual consiste en la superposición de dos rejillas (denominadas Kp y $\mathrm{Kg}$ ) para cuantificar los espacios de ocupación para cada uno de los atractores. Se aplicará la ley exponencial( ${ }^{(22)}$ para la evaluación diagnóstica. Posteriormente se aplicó la metodología de evaluación matemática al análisis de la totalidad del registro para cada holter y registro electrocardiográfico continuo, en busca de comparaciones entre esta evaluación y la obtenida en 18 horas.

La ley exponencial asocia la enfermedad aguda a espacios de ocupación Kp inferiores a 73. Un diagnóstico físico-matemático de normalidad se establecerá cuando se encuentren espacios de ocupación Kp mayores de 200. Y la evolución es cuantificada con valores que se encuentren entre 73 y 200.

Finalmente la evaluación obtenida en 18 horas será comparada con el diagnóstico clínico convencional hallando su concordancia diagnóstica con el coeficiente Kappa, y calculando sensibilidad y especificidad.

\section{Análisis estadístico}

Los resultados hallados al aplicar esta metodología a 18 horas, fueron comparados con el diagnóstico clínico convencional, respecto al Gold estándar. Para esto se hizo una clasificación binaria, en la que los verdaderos positivos (VP) son los casos evaluados como patológicos tanto matemáticamente como convencionalmente; falsos positivos (FP) son los casos que fueron diagnosticados convencionalmente como normales por parte del experto, pero con evaluación matemática de enfermedad; falsos negativos (FN) son los casos evaluados matemáticamente como normales pero convencionalmente como enfermos; verdaderos negativos (VN) son los casos diagnosticados convencionalmente y matemáticamente como normales. Se evaluó la concordancia entre el diagnóstico convencional y el físico-matemático por medio de la ecuación del coeficiente Kappa:

$$
K=\frac{C o-C a}{T o-C a}
$$

En donde, Co son las concordancias observadas entre el diagnóstico desde la metodología matemática y desde el Gold estándar; To representa la totalidad de casos; Ca son las concordancias atribuibles al azar que se calculan a través de la siguiente ecuación:

$$
C a=\left[\left(f_{1} x C_{1}\right) / T o\right]+\left[\left(f_{2} x C_{2}\right) / T o\right]
$$

Donde $f_{1}$ es el número de casos los casos evaluados matemáticamente como normales; $\mathrm{C}_{1}$ es el número de casos evaluados convencionalmente diagnosticados como normales; $f_{2}$ representa el número de casos evaluados matemáticamente como enfermedad; $\mathrm{C}_{2}$ representa el número de casos diagnosticados convencionalmente como patológicos; $T_{0}$ representa el número total de casos.

\section{Aspectos éticos}

Esta investigación se adhiere a los principios éticos de la Declaración de Helsinki de la Asociación Médica Mundial. Según el artículo 11 de la resolución 008430 de 1993, del Ministerio de salud, el tipo de riesgo inherente a la investigación correspondería a investigación con riesgo mínimo, al estar clasificado en la categoría de investigación sin riesgo, pues se hacen cálculos físicos y matemáticos sobre resultados de exámenes de la práctica clínica que han sido prescritos médicamente, protegiendo la integridad y anonimato de las participantes. Se hace aclaración que se cumple con el artículo 13 de ésta misma resolución ${ }^{(24)}$. 


\section{Resultados}

Las dimensiones fractales (tabla 1) de los registros Holter evaluados en mínimo 21 horas con diagnóstico clínico normal variaron entre 1,1316 y 1,9122 mientras que para los registros con alguna patología variaron entre 1,0198 y 1,9532. Los espacios ocupados por los atractores normales evaluados mínimo en 21 horas, al superponer la rejilla Kp oscilaron entre 200 y 381 , para la rejilla $\mathrm{Kg}$ entre 55 y 147; para los pacientes enfermos la superposición de la rejilla $\mathrm{Kp}$, oscilo entre 35 y 187; y entre 9 y 83 para la rejilla $\mathrm{Kg}$ (tabla 1).

Tabla 1. Medidas de atractores de algunos de los holter estudiados.

\begin{tabular}{|c|c|c|c|c|c|c|c|}
\hline \multirow[b]{2}{*}{$\begin{array}{l}\text { Holter } \\
\text { No. }\end{array}$} & \multirow[b]{2}{*}{ Indicaciones } & \multicolumn{3}{|c|}{21 horas } & \multicolumn{3}{|c|}{18 horas } \\
\hline & & Kp & $\mathbf{K g}$ & Df & $\mathbf{K p}$ & Kg & Df \\
\hline 1 & Normal & 293 & 105 & 1,4805 & 294 & 107 & 1,4582 \\
\hline 2 & $\begin{array}{l}\text { Episodio de } \\
\text { síncope } \\
\text { vasovagal }\end{array}$ & 137 & 39 & 1,8126 & 133 & 40 & 1,7334 \\
\hline 3 & $\begin{array}{l}\text { Taquicardia - } \\
\text { ventricular }\end{array}$ & 117 & 35 & 1,7411 & 120 & 37 & 1,6974 \\
\hline 4 & Normal & 279 & 91 & 1,6163 & 275 & 89 & 1,6276 \\
\hline 5 & Normal & 217 & 67 & 1,6955 & 220 & 68 & 1,6939 \\
\hline 6 & $\begin{array}{l}\text { TAM, } \\
\text { insuficiencia } \\
\text { respiratoria }\end{array}$ & 156 & 51 & 1,6130 & 160 & 51 & 1,6495 \\
\hline 7 & $\begin{array}{l}\text { SCA, Infarto } \\
\text { del miocardio } \\
\text { con elevación } \\
\text { ST }\end{array}$ & 65 & 31 & 1,0682 & 62 & 33 & 0,9098 \\
\hline 8 & $\begin{array}{l}\text { Palpitaciones, } \\
\text { Síndrome } \\
\text { Wolff- } \\
\text { Parkinson- } \\
\text { White } \\
\end{array}$ & 35 & 9 & 1,9594 & 38 & 10 & 1,9250 \\
\hline 9 & Normal & 285 & 104 & 1,4544 & 280 & 103 & 1,4428 \\
\hline 10 & Normal & 218 & 92 & 1,2446 & 217 & 91 & 1,2538 \\
\hline 11 & Normal & 202 & 58 & 1,8002 & 205 & 58 & 1,8215 \\
\hline 12 & Normal & 271 & 73 & 1,8923 & 269 & 72 & 1,9015 \\
\hline 13 & Normal & 315 & 96 & 1,7142 & 318 & 95 & 1,7430 \\
\hline 14 & Normal & 372 & 144 & 1,3692 & 377 & 145 & 1,3785 \\
\hline 15 & Normal & 331 & 116 & 1,5127 & 332 & 117 & 1,5047 \\
\hline 16 & Normal & 348 & 109 & 1,6748 & 344 & 108 & 1,6714 \\
\hline 17 & $\begin{array}{l}\text { sincope, } \\
\text { disminucion } \\
\text { moderada de la } \\
\text { variabilidad de } \\
\text { la FC, EV } \\
\text { infrecuente } \\
\text { monomorfica } \\
\text { sin fenomenos } \\
\text { repetitivos }\end{array}$ & 158 & 47 & 1,7492 & 156 & 45 & 1,7935 \\
\hline 18 & Normal & 270 & 93 & 1,5377 & 273 & 93 & 1,5536 \\
\hline 19 & $\begin{array}{l}\text { Defecto } \\
\text { cardíaco } \\
\text { congénito - } \\
\text { CIA } \\
\end{array}$ & 151 & 49 & 1,6237 & 152 & 50 & 1,6041 \\
\hline 20 & $\begin{array}{l}\text { palpitaciones, } \\
\text { mareo, }\end{array}$ & 165 & 67 & 1,3002 & 160 & 66 & 1,2775 \\
\hline
\end{tabular}

\begin{tabular}{|c|c|c|c|c|c|c|c|}
\hline & taquicardia & & & & & & \\
\hline 21 & $\begin{array}{l}\text { fibriloaleteo } \\
\text { auricular, } \\
\text { fatiga, sincope }\end{array}$ & 88 & 23 & 1,9359 & 88 & 23 & 1,9359 \\
\hline 22 & Normal & 261 & 77 & 1,7611 & 261 & 76 & 1,7800 \\
\hline 23 & $\begin{array}{l}\text { FA, Dificultad } \\
\text { respiratoria }\end{array}$ & 173 & 51 & 1,7622 & 169 & 53 & 1,6730 \\
\hline 24 & Normal & 268 & 75 & 1,8373 & 273 & 77 & 1,8260 \\
\hline 25 & $\begin{array}{l}\text { Sensación de } \\
\text { percibir los } \\
\text { latidos } \\
\text { cardíacos } \\
\text { (palpitaciones) }\end{array}$ & 179 & 75 & 0 & 183 & 74 & 1,3062 \\
\hline 26 & $\begin{array}{l}\text { Fibriloaleteo } \\
\text { auricular, } \\
\text { dificultad } \\
\text { respiratoria }\end{array}$ & 162 & 52 & 1,6394 & 161 & 51 & 1,6585 \\
\hline 27 & $\begin{array}{l}\text { Arritmia en } \\
\text { estudio }\end{array}$ & 145 & 41 & 1,8224 & 151 & 42 & 1,8460 \\
\hline 28 & $\begin{array}{l}\text { Desmayo, } \\
\text { fibrilacion } \\
\text { ventricular }\end{array}$ & 59 & 22 & 1,4232 & 55 & 23 & 1,2578 \\
\hline 29 & $\begin{array}{l}\text { Taquicardia } \\
\text { auricular } \\
\text { multifocal }\end{array}$ & 158 & 77 & 1,0370 & 162 & 78 & 1,0544 \\
\hline 30 & Flutter & 38 & 14 & 1,4406 & 37 & 15 & 1,3026 \\
\hline 31 & Normal & 262 & 84 & 1,6411 & 262 & 84 & 1,6411 \\
\hline 32 & Palpitaciones & 170 & 76 & 1,1615 & 172 & 75 & 1,1974 \\
\hline
\end{tabular}

En cuanto a los registros evaluados en 18 horas mediante la presente metodología, las dimensiones fractales (tabla 1 ) de los registros Holter con diagnóstico clínico normal se encontraron entre 1,2019 y 1,9207; y entre 0,8961 y 1,9431 para los holter de pacientes con alguna patología. Los espacios ocupados por los atractores normales al superponer la rejilla $\mathrm{Kp}$ oscilaron entre 201 y 384, y para la rejilla $\mathrm{Kg}$ entre 56 y 147; para los casos con alguna patología los valores ocupados con la superposición de la rejilla Kp, osciló entre 37 y 379, y para la rejilla Kg entre 10 y 147.

(a)

(b)

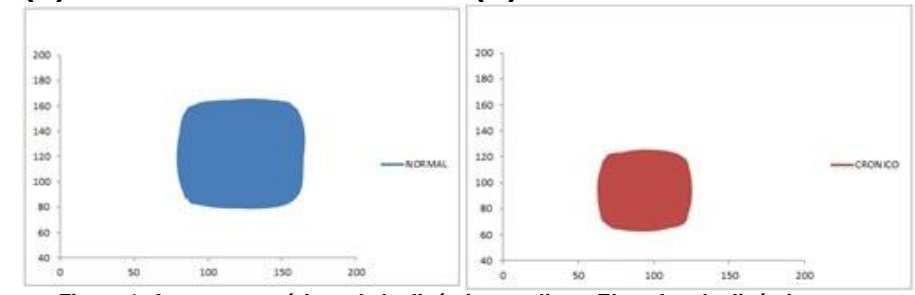

Figura 1. Atractores caóticos de la dinámica cardiaca. Ejemplos de dinámicas normal (a) y crónica (b); corresponden a los holter No. 24 y 27 respectivamente de la tabla 1. 


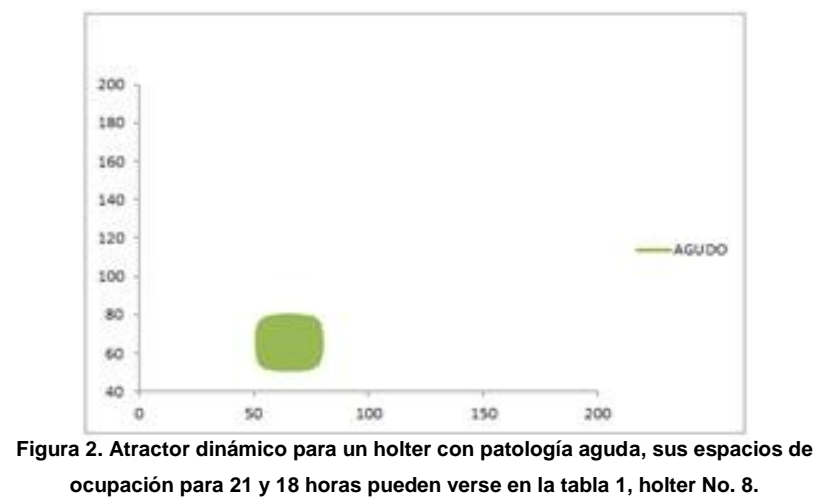

Se encontraron valores de sensibilidad y especificidad del $100 \%$, y un coeficiente Kappa igual a 1, entre la metodología diagnostica convencional y la evaluación diagnóstica con la ley matemática aplicada en 18 horas. La metodología diferencia de manera adecuada los casos de normalidad y los casos que corresponden a estados de patología aguda, también es posible observar como el decremento de espacios de ocupación de los atractores puede indicar la evolución hacia estados patológicos o agudos de la dinámica cardiaca.

Tabla 1. Medidas de atractores de algunos de los holter estudiados.

\section{Discusión}

Este es el primer trabajo en el que la ley exponencial de la dinámica cardiaca caótica aplicada en 18 horas es aplicada a la evaluación de 120 registros Holter y electrocardiográficos continuos normales y con diferentes patologías, cuantificando los espacios de ocupación de los atractores de las dinámicas; se encontraron diferencias objetivas y reproducibles entre las dinámicas estudiadas, tanto en la aplicación en 18 horas, como cuando se evaluó todo el registro. Al comparar ambas evaluaciones se encontró que fueron concordantes para cada registro, de igual forma se pudo observar como la metodología logra evaluar cuantitativamente diferentes grados de anormalidad en la dinámica cardiaca aun en menos tiempo de evaluación.

Esta investigación está sustentada en el método de la física teórica ${ }^{(25,26)}$, por esto es posible evidenciar un orden matemático subyacente al proceso de normalidad-enfermedad de la dinámica cardiaca, al margen de la etiología de la patología, caracterizado por una variación progresiva del número de espacios ocupados por el atractor caótico. La auto-organización matemática evidenciada es aplicable a cualquier caso particular, sin tener en cuenta parámetros estadísticos 0 epidemiológicos. Esta autoorganización matemática del fenómeno es la que permite establecer una evaluación en menos tiempo que sea consistente con la evaluación desarrollada para la totalidad del registro.

La ley exponencial aplicada en este trabajo, ha permitido deducir y evaluar todas las posibles dinámicas cardiacas, caracterizando dinámica cardiacas normales, agudas y en evolución ${ }^{(22)}$. De igual forma la metodología evaluó la dinámica cardiaca neonatal, logrando predecir alteraciones cardiacas asociadas a sepsis tres y seis horas antes de presentarse el episodio de sepsis ${ }^{(27)}$. Una prueba diagnóstica de esta metodología evaluó la aplicabilidad clínica y la reproductibilidad mediante un estudio ciego con 115 registros Holter ${ }^{(23)}$; también fue confirmada su aplicabilidad a casos con diagnóstico de $\operatorname{arritmia}^{(24)}$. La aplicación de la metodología desarrollada en este trabajo constituye una herramienta de ayuda diagnostica del paciente en menos tiempo, lo que puede alertar de manera temprana sobre la aparición episodios agudos.

Mediante la electrocardiografía, las señales eléctricas del corazón son reproducidas de manera gráfica, esta es una herramienta ampliamente utilizada para detectar alteraciones en el ritmo cardiaco ${ }^{(29)}$. Sin embargo desde estas herramientas aún no es posible predecir cual dinámica cardiaca evolucionará a algún tipo de complicación aguda. Múltiples trabajos en los cuales se estudia la Variabilidad de la Frecuencia Cardiaca (VFC) se han desarrollado(15-19), la aplicabilidad clínica de estas metodologías es discutida ${ }^{(20)}$, o se necesitan más estudios para confirmarlas $^{(21)}$. La metodología aplicada para el presente estudio, puede diagnosticar cualquier caso particular, a partir de la ocupación espacial del atractor en el contexto de una ley, permitiendo un seguimiento más estricto de los procesos de la dinámica cardiaca del paciente en el tiempo a partir de los atractores cardiacos.

La geometría fractal ha sido aplicada en diferentes ámbitos de la medicina ${ }^{(30-35)}$ en busca de diferenciar normalidad de enfermedad, sin embargo, como se muestra en el presente trabajo no son las dimensiones fractales las que permiten establecer esta diferenciación, específicamente para la dinámica cardiaca la diferencia entre los espacios de ocupación del atractor $^{(22,23,28)}$ es lo que permite la evaluación diagnostica de cada caso particular, independientemente de metodologías estadísticas y epidemiológicas que describen comportamientos poblacionales. 
Desde la perspectiva de la aplicación de teorías físico-matemáticas a la medicina se han logrado diagnósticos y predicciones objetivas $y$ reproducibles en áreas como la morfología ${ }^{(36,37)}$, en inmunología ${ }^{(38)}$, en infectología ${ }^{(39)}$, biología molecular ${ }^{(40)}$, así como en cardiología ${ }^{(41-43)}$ o salud pública, donde se desarrolló un método de predicción de brotes de malaria en 820 municipios de Colombia en períodos de tres semanas $^{(44)}$, con una exactitud del $99.86 \% 51$. También se desarrollaron predicciones de mortalidad de la unidad de cuidados intensivos ${ }^{(45)}$ a partir de la teoría de los sistemas dinámicos y la teoría de conjuntos.

\section{Conclusión}

Se demostró que la ley exponencial en la dinámica cardiaca caótica asociada a arritmias permite la aplicabilidad clínica en 18 horas para la evaluación de 120 registros Holter y electrocardiográficos continuos normales y con diferentes patologías permitiendo la predicción de la evolución hacia estados agudos de la dinámica.

\section{Dedicatoria}

A nuestros hijos

\section{Agradecimientos}

Agradecemos al Hospital Universitario Mayor Méderi, por su apoyo a nuestras investigaciones. También extendemos nuestros agradecimientos al Centro de Investigaciones de la Clínica del Country por apoyo a nuestras investigaciones; a los Doctores Tito Tulio Roa, Director de Educación Médica, Jorge Ospina, Director Médico, Alfonso Correa, Director del Centro de Investigaciones, y a las Doctoras Adriana Lizbeth, epidemióloga y Silvia Ortiz, Enfermera Jefe del Centro de Investigaciones, así como a Sandra Rodríguez, enfermera del Centro.

\section{Bibliografía}

1. Devaney R. A first course in chaotic dynamical systems theory and experiments. Reading Mass: Addison-Wesley 1992.

2. Peitgen $H$, Jürgens $H$, Saupe $D$. Strange attractors, the locus of chaos. En: Chaos and Fractals: New Frontiers of Science. New York: Springer-Verlag, 1992, pp. 655-768.

3. Goldberger AL, Bhargava V, West BJ, Mandell AJ. Some observations on the question: Is ventricular fibrillation "chaos?" Physica D 1986;19:282-9.

4. Mandelbrot B. Cambios de escala y leyes potenciales sin geometría. En: The Fractal Geometry of Nature. San Francisco: Freeman Ed., 1972, pp. 477-87.
5. Mandelbrot B. Árboles jerárquicos o de clasificación, y la dimensión. En: Los Objetos Fractales. Tusquets Eds S.A. Barcelona. 2000: 161-6.

6. Mandelbrot B. How Long Is the Coast of Britain? Statistical Self-Similarity and Fractional Dimension. Science 1967; 156: 636-38.

7. OMS- Centro de prensa. Día mundial del corazón: Enfermedades Cardiovasculares causan 1,9 millones de muertes al año en las Américas. Nota informativa octubre de 2012.2 En: http://www.paho.org/bol/index.php?option=com content \&view=article\&id=1514\&catid=667: notas-de-prensa.

8. Mozaffarian D, Benjamin E, Go AS, Arnett DK, Blaha MJ, et al. Heart Disease and Stroke Statistics-2015 Update. A Report From the American Heart Association. Circulation 2015; 2015;131:e29-e322.

9. Barron H, Viskin S. Autonomic markers and prediction of cardiac death after myocardial infarction. Lancet 1998; 351: $461-2$.

10. Kouchoukos NT. Kirklin/Barratt-Boyes Cardiac Surgery: Morphology, Diagnostic Criteria, Natural History, Techniques, Results, and Indications. Michigan: Churchill Livingstone, 2003.

11. Gómez $M$, Enciso $C$, Peña $E$, Segura $O D$, Nieto $V H$. Ecais study: inadvertent cardiovascular adverse events in sepsis. Med Intensiva 2012; 36(5):343-350

12. Goldberger A, Amaral L, Hausdorff JM, Ivanov P, Peng Ch, Stanley HE. Fractal dynamics in physiology: alterations with disease and aging. PNAS 2002; 99: 2466 - 2472

13. Higgins JP. "Nonlinear systems in medicine". Yale. J. Biol. Med. 2002; 75: 247-60.

14. Costa M, Goldberger AL, Peng CK. Multiscale Entropy Analysis of Complex Physiologic Time Series. Phys Rev Lett. 2002;89 (6): 0681021 - 4.

15. Wu GQ, Arzeno NM, Shen LL, Tang DK, Zheng DA, et al. Chaotic Signatures of Heart Rate Variability and Its Power Spectrum in Health, Aging and Heart Failure. PLoS ONE. (2) 4 (2009), e4323.

16. Braun $C$, Kowallik $P$, Freking $A$, Hadeler $D$, Kniffki $K$, Meesmann M. Demonstration of nonlinear components in heart rate variability of healthy persons Am. J. Physiol. 1998; 275, H1577-H1584.

17. Porta A, Guzzetti S, Montano N, Furlan R, Pagani M, et al. 2001 Entropy, entropy rate and pattern classification as tools to typify complexity in short heart period variability series. IEEE Trans. Biomed. Eng. 48, 12821291.

18. Guzzetti S, et al. 2005 Symbolic dynamics of heart rate variability: a probe to investigate cardiac autonomic modulation. Circulation 112, 465-470.

19. Maestri R, Pinna GD, Accardo A, Allegrini P, Balocchi $R$, et al. Nonlinear indices of heart rate variability in chronic heart failure patients: redundancy and comparative clinical value. J. Cardiovasc. Electrophysiol. 2007; 18: 425-433.

20. Voss A, Schulz S, Schroeder R, Baumert M, Caminal P, Methods derived from nonlinear dynamics for analysing heart rate variability. Philos Trans A Math Phys EngSci. 2009; 367:277-96.

21. J. Perkiömäki TH, Mäkikallio HV, Huikuri, Fractal and Complexity Measures of Heart Rate Variability. Clin. Exp. Hypertens. 2005; 2(3):149-158.

22. Rodríguez J. Mathematical law of chaotic cardiac dynamic: Predictions of clinic application. J Med Med Sci. 2011; 2(8):1050-1059.

23. Rodríguez J, Correa C, Melo M, Domínguez D, Prieto $S$, et al. Chaotic cardiac law: Developing predictions of clinical application. J. Med. Med. Sci. 2013;4(2): 79-84. 
24. Colombia. Ministerio de salud. Resolución número 8430. Por la cual se establecen las normas científicas, técnicas y administrativas para la investigación en salud. Bogotá D.C.;1993.

25. Einstein $A$. Sobre la teoría de la relatividad y otras aportaciones científicas. Madrid: Sarpe, 1983, pp. 2332.

26. Einstein $A$. Sobre la teoría de la relatividad y otras aportaciones científicas. Madrid: Sarpe, 1983, pp. 7884.

27. Rodríguez J, Prieto S, Flórez M, Alarcón C, López R, et al. Physical-mathematical diagnosis of cardiac dynamic on neonatal sepsis: predictions of clinical application. J. Med. Med. Sci 2014; 5(5): 102-108.

28. Rodríguez J, Prieto $S$, Domínguez D, Correa C, Melo M, et al. Application of the chaotic power law to cardiac dynamics in patients with arrhythmias. Rev. Fac. Med. 2014;62(4):539-46.

29. Pineda M, Matiz $H$, Rozo R. Enfermedad coronaria. Bogotá: Editorial Kimpres Ltda., 2002.

30. Dey $P$, Rajesh L. Fractal dimesion in endometrial carcinoma. Anal Quant Cytol Histol 2004;26(2):113-6.

31. Garte S. Fractal properties of the human genome. J Theor Biol 2004; 230(2):251-60.

32. Kikuchi A, Kozuma S, Yasugi T, Taketani Y. Fractal analysis of the suface growth patterns in endometrioid endometrial adenocarcinoma. Gynecol Obstet Invest 2004;58(2):61-7.

33. Zatloukal Z. Granulometry and fractal dimensions. Ceska Slov Farm 2003; 52(5):244-7.

34. Gazit Y, Baish JW, Safabaksh N. Fractal characteristics of tumour vascular architecture during tumour growth and regression. Microcirculation. 1997; 395-402.

35. Lefebvre F, Benali HA. Fractal approach to the segmentation of microcalcifications in digital mammograms. Med. Phys. 1995; 22: 381 - 390.

36. Rodríguez J, Prieto S, Correa C, Bernal P, Puerta G, et al. Theoretical generalization of normal and sick coronary arteries with fractal dimensions and the arterial intrinsic mathematical harmony. BMC Med Phys 2010; 10: 1-6.

37. Prieto $S$, Rodríguez J, Correa C, Soracipa Y. Diagnosis of cervical cells based on fractal and Euclidian geometrical measurements: Intrinsic Geometric Cellular Organization. BMC Medical Physics 2014, 14(2): 1-9.

38. Rodríguez J. Teoría de unión al HLA clase II teorías de Probabilidad Combinatoria y Entropía aplicadas a secuencias peptídicas. Inmunología. 2008; 27(4): 151166.

39. Rodríguez J, Prieto S, Correa C, Pérez C, Mora J, et al. Predictions of CD4 lymphocytes' count in HIV patients from complete blood count. BMC Medical Physics. BMC Medical Physics. 2013; 13: 1-3.

40. Rodríguez J, Bernal P, Prieto S, Correa C. Teoría de péptidos de alta unión de malaria al glóbulo rojo. Predicciones teóricas de nuevos péptidos de unión y mutaciones teóricas predictivas de aminoácidos críticos. Inmunología. 2010; 29(1): 7-19.

41. Rodríguez J, Prieto S, Correa C, Bautista J, Velasco A, et al. Mathematics physical assessment of cardiac dynamics based on theory of probability and proportions of entropy in the Intensive care Unit for patients with arrhythmia. International Conference on Medical Phyiscs. Aug. 3-5 2015. Birmingham UK. J Nucl Med Radiat Ther. 2015;6:4

42. Rodríguez J, Prieto $S$, Bernal $P$, Izasa D, Salazar G, et al. Entropía proporcional aplicada a la evolución de la dinámica cardiaca. Predicciones de aplicación clínica. En: Rodríguez LG, Coordinador. La emergencia de los enfoques de la complejidad en América Latina: implicancias políticas, epistemológicas y metodológicas para las ciencias del siglo XXI. Tomo 1,1a ed. Buenos Aires: Comunidad Editora Latinoamericana; 2015. p. 247-64.

43. Rodríguez J, Prieto S, Correa C, Mendoza F, Weiz G, et al. Physical mathematical evaluation of the cardiac dynamic applying the Zipf - Mandelbrot law. Journal of Modern Physics 2015, 6: 1881-1888. http://dx.doi.org/10.4236/jmp.2015.613193

44. Rodríguez J. Método para la predicción de la dinámica temporal de la malaria en los municipios de Colombia. Rev Panam Salud Pública. 2010; 27(3): 211-218.

45. Rodríguez J. Dynamical systems applied to dynamic variables of patients from the intensive care unit (ICU): Physical and mathematical mortality predictions on ICU. J. Med. Med. Sci. 2015; 6(8):209-220. 